\title{
Temporal study of countries' scientific evolution based on two-dimensional approach
}

\author{
Seyyed Mehdi Hosseini Jenab \\ Department of Physics, South Tehran Branch, Islamic Azad University, Tehran, Iran
}

\begin{abstract}
Evolution of 50 top most countries in scientific production is analyzed over 16 years (1996-2011) based on two-dimensional approach, that is, considering both quantity and quality simultaneously. By applying an averaging method, the global trend both in paper publication as quantity indicator and their citations as a quality indicator have been removed. Three different patterns of temporal evolution are detected among these countries in the two-dimensional map out of five possible ones. 11 countries are revealed to have considerable progress rate. Their temporal evolution is studied and they are ranked based on their achievement in the study period. Furthermore, eight countries are found to show considerable decline rate, and 30 countries follow the fluctuation pattern. China and USA show the fastest progress and decline rate respectively.
\end{abstract}

Keywords: Countries' scientific evolution, two-dimensional mapping, quality-quantity approach

\section{INTRODUCTION}

Temporal evolution of countries' scientific achievement is mostly analyzed based on the single-indicator method in which different aspects of scientific activity are investigated individually. ${ }^{[1-4]}$ This method results in various rankings and set of tables, which mostly fail to provide decisive picture; furthermore, this method isn't able to show the relationship among different aspects properly. Hence, other methods such as a composite indicators ${ }^{[5,6]}$ and two-dimensional approach ${ }^{[7,8]}$ have been proposed in recent years to solve these issues.

The two-dimensional approach is inspired by Hirsch in his paper about h-index. ${ }^{[9]}$ Hirsch method is based on considering both quantity (publication numbers) and

*Address for correspondence:

E-mail: mehdi.jenab@yahoo.com

\begin{tabular}{|l|l|}
\hline \multicolumn{2}{|c|}{ Access this article online } \\
\hline Quick Response Code: & \\
\hline & Website: \\
\hline & www.jscires.org \\
\cline { 2 - 3 } & \\
\hline
\end{tabular}

quality (citation numbers) of an individual researcher simultaneously and provides one single number for the assessment. However, h-index isn't designed to analyze the temporal evolution because of losing chronological order in drawing the so-called h-index diagram.

The two-dimensional approach tries to avoid segregated set of indicators and consider both indicators at the same time. This method provides a two-dimensional map, which save the chronological order and hence the temporal evolution can be studied properly based on this method. Numbers used in the map should be comparable to each other in order to provide a fair visual judgment; this main concern is met by using modified version of publication and citation numbers.

Single-indicators like paper publication per year or citation results in a sharp ranking of countries, which is to some extent meaningless since nearby numbers in these indicators can't be decisive in the ranking. But in two-dimensional approach one can distinguish these vicinities in the map and be able to avoid misjudgment based on visual judgment provided by this method.

Here, the focus is on the temporal evolution of the first 50 countries in paper publication over the 16 years (1996-2011) in the two-dimensional map. Since 1996, the scientific 
activity intensity has grown rapidly, ${ }^{[10]}$ and this reflects on the national level. However the pattern of growth for all countries can't be the same and this study shows the patterns, which countries have been following for the last 16 years. One must take into account that progress in quantity and quality of scientific activity are comparatively independent from each other. Therefore by considering both quantity and quality of national scientific activity, different pattern of temporal evolution will emerge.

\section{MATERIALS AND METHODS}

Publication number (represented here by $P$ ) presents the number of articles of each country in every year published in peer reviewed journals and it is considered as the quantity indicator of country's scientific activity. For quality indicator, citation number (represented here by $C$ ) is chosen which presents the number of citations that papers, published in the same year, received from their publication year up to 2011. These two indicators ( $P$ and $C$ ) have been considered as the most important base for analyzing quantity and quality of scientific achievement of countries in many national and international reports. ${ }^{[1-4]}$ Publication number depends on two variables, namely, year of publication $\left(y_{j}\right)$ and country $\left(c_{j}\right)$, in which parameter i runs from 1 to 16, since the period of 16 years is covered in this study. Parameter $j$ ranges from 1 to 50 , because 50 countries are considered here. For citation the two variables are citation window $\left(\mathrm{y}_{\mathrm{i}}-\mathrm{y}_{10}\right)$ and country $\left(\mathrm{c}_{\mathrm{j}}\right)$. For example, the publication number of The Netherlands in 2002 is $P(2002$, Netherlands $)=P\left(\mathrm{y}_{7}, \mathrm{c}_{11}\right)=23813$ and the related citation number is and the related citation number is $C$ (2002-2011, Netherlands) $=P\left(\mathrm{y}_{7}-\mathrm{y}_{16}, \mathrm{c}_{11}\right)=635188$. Data for this study is extracted from scimagojr, which is based on Scopus database. ${ }^{[1]}$

Figure 1 shows the map based on the pair of $P$ and $C$ for different countries over 16 years. Since each paper needs a few years after its publication to be viewed in the scientific community and to be cited, so a downfall trend can be observed in the all-time lines as they approach the recent years. This trend twisted the time lines so strongly that the map isn't any more useful or understandable. Furthermore, $P$ and $C$ for different years isn't comparable since the context of each year is different due to global rapid progress in scientific activity during these 16 years (actually publication numbers of the world has doubled)..$^{[10]}$

In order to resolve these issues, normalization method is applied which changes these numbers into dimensionless numbers through dividing them by an average. The average

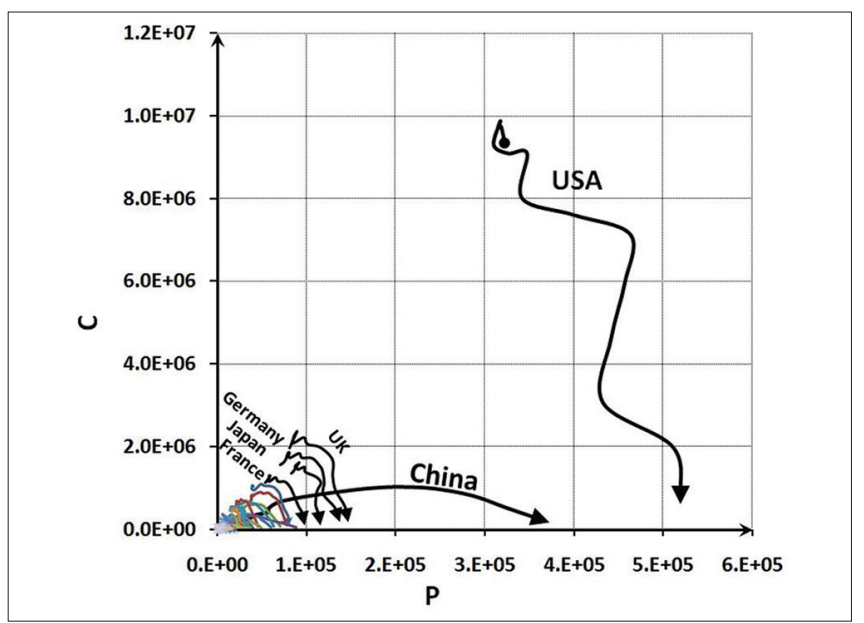

Figure 1: Two-dimensional map based on $\mathrm{P}$ (publication number) and $\mathrm{C}$ (citation number). Decreasing citation numbers of recent years twist time lines for different countries and makes the map useless in analyzing temporal evolution of countries

is over all countries publication or citation numbers in each year. Therefore, $\mathrm{Pm}$ (modified publication number) and $\mathrm{Cm}$ (modified citation number) are introduced as follow:

$$
\begin{aligned}
& P m\left(\mathrm{y}_{\mathrm{i}}, \mathrm{c}_{\mathrm{j}}\right)=\frac{P\left(\mathrm{y}_{\mathrm{i}}, \mathrm{c}_{\mathrm{j}}\right)}{\frac{1}{50} \sum_{\mathrm{j}=1}^{50} P\left(\mathrm{y}_{\mathrm{i}}, \mathrm{c}_{\mathrm{j}}\right)}, \\
& \operatorname{Cm}\left(\mathrm{y}_{\mathrm{i}}, \mathrm{c}_{\mathrm{j}}\right)=\frac{C\left(\mathrm{y}_{\mathrm{i}}-\mathrm{y}_{16}, \mathrm{c}_{\mathrm{j}}\right)}{\frac{1}{50} \sum_{\mathrm{j}=1}^{50} C\left(\mathrm{y}_{\mathrm{i}}-\mathrm{y}_{16}, \mathrm{c}_{\mathrm{j}}\right)}
\end{aligned}
$$

Since these numbers are dimensionless, so they can be compared over different years.

For example, $P \mathrm{~m}(2002$, Netherlands $)=\left(\mathrm{y}_{7}, \mathrm{c}_{11}\right)=0.96$ shows that Netherlands in year 2002 has publication 0.96 times the publication average of the world in that year.

The two-dimensional map is sketched by assigning $\mathrm{x}$-axis to $P \mathrm{~m}$ and y-axis to $C \mathrm{~m}$. Each pair of $P \mathrm{~m}$ and $C \mathrm{~m}$ associated with a specific year represented as one point in the map. Hence, every country has 16 points representing each year from 1996 up to 2011. By connecting these points together chronologically, a temporal line appears for each country, showing its temporal evolution [Figure 2].

Basically, these temporal lines can be categorized into five different patterns [Figure 3], which are combinations of progress or decline in either $P \mathrm{~m}$ or $\mathrm{Cm}$ :

- Progress in both $P \mathrm{~m}$ and $C \mathrm{~m}$,

- Progress in $P \mathrm{~m}$ and decline in $\mathrm{Cm}$,

- Decline in $P \mathrm{~m}$ and progress in $\mathrm{Cm}$, 
- Decline in both $P \mathrm{~m}$ and $C \mathrm{~m}$,

- Fluctuation; which means that time line can be comprehended as just fluctuation around a certain position in the map and countries with displacement in both axes less than a certain threshold are considered as fluctuation.

\section{RESULTS AND DISCUSSION}

Eleven countries are shown to have progress pattern, and can be divided into three subgroups based on their displacement in the map as follow:

- China [Figures 2 and 4]

- Group A: India, South Korea, Iran, Brazil,Turkey [Figure 5]

- Group B: Malaysia, Taiwan, Spain, Singapore,Australia [Figure 6].

China with wide shift in its placement in the map presents an outstanding achievement which makes it an exception

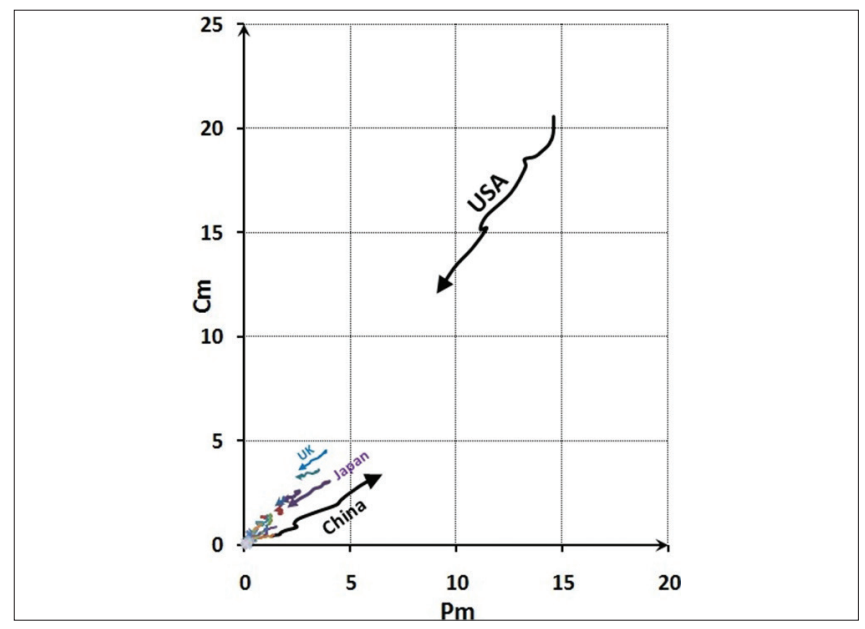

Figure 2: Two-dimensional map for all 50 countries. The decline pattern of USA and progress pattern of China stand out

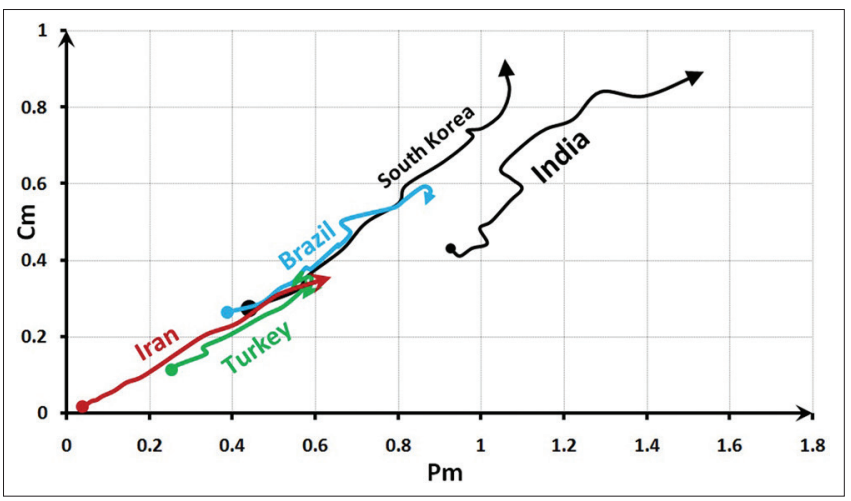

Figure 4: Two-dimensional map of Group A of progress pattern. Members of this group have shown wide range of change in their position in the map during 1996-2011 from other members of progress pattern. Group A members possess a wide range of change and present a leap of progress in their time lines. Countries in Group B achieved rather smaller change in their position in the map.

No country has the pattern of "progress in $P \mathrm{~m}$ and decline in $C \mathrm{~m}$ " which is to some extent expectable, since progress in $\mathrm{Pm}$ mostly likely results in progress in $\mathrm{Cm}$. However, Italy has shown interesting temporal evolution, it is the only country with the pattern of "decline in $P \mathrm{~m}$, but progress in Cm" [Figure 4].

A total of 30 countries have shown no considerable change in their positions in the map during the studied time period so they are labeled as "fluctuation," which means their

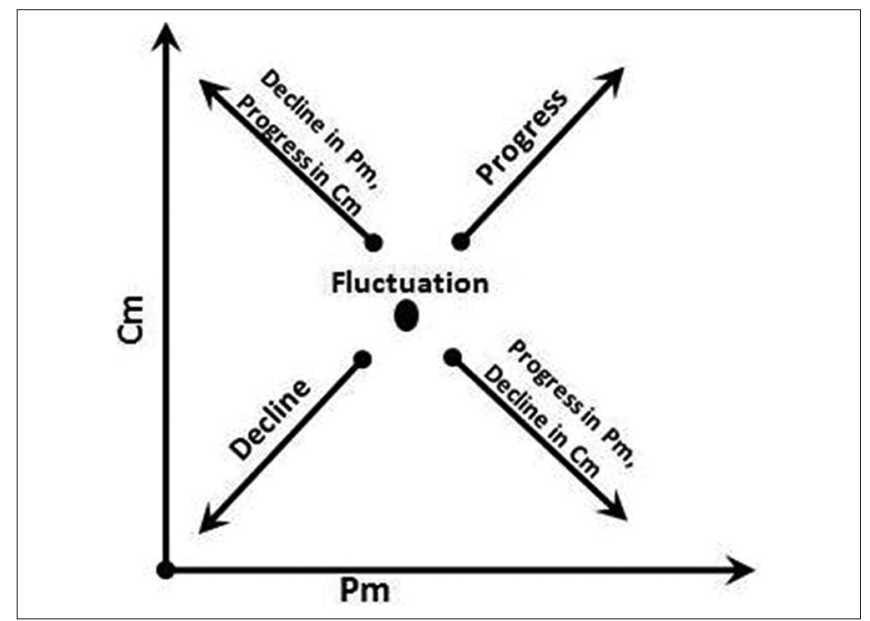

Figure 3: Different patterns in the two-dimensional map. Five patterns exist based on the combination of progress and decline over each of two axis

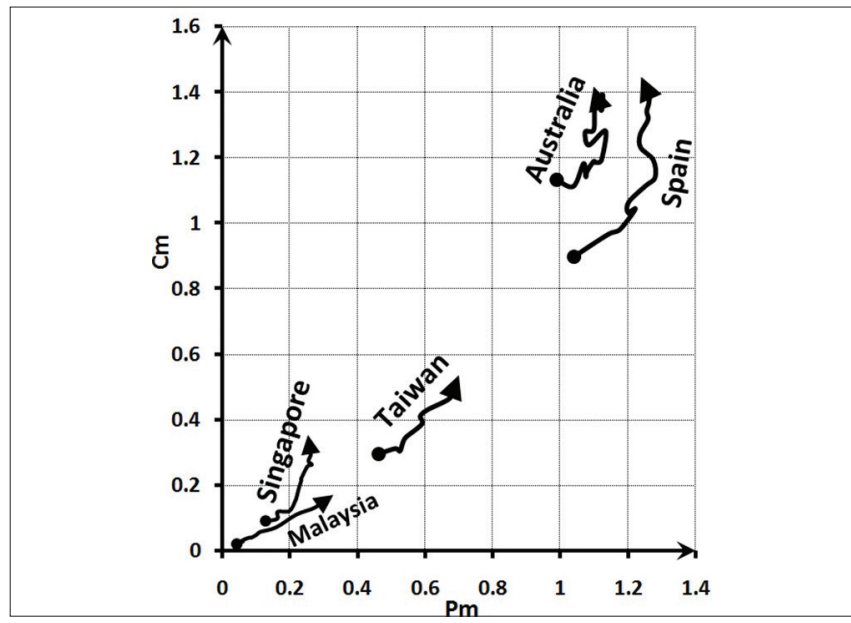

Figure 5: Two-dimensional map of Group B of progress pattern. Members of this group has shown change in their position in the map during 1996-2011 


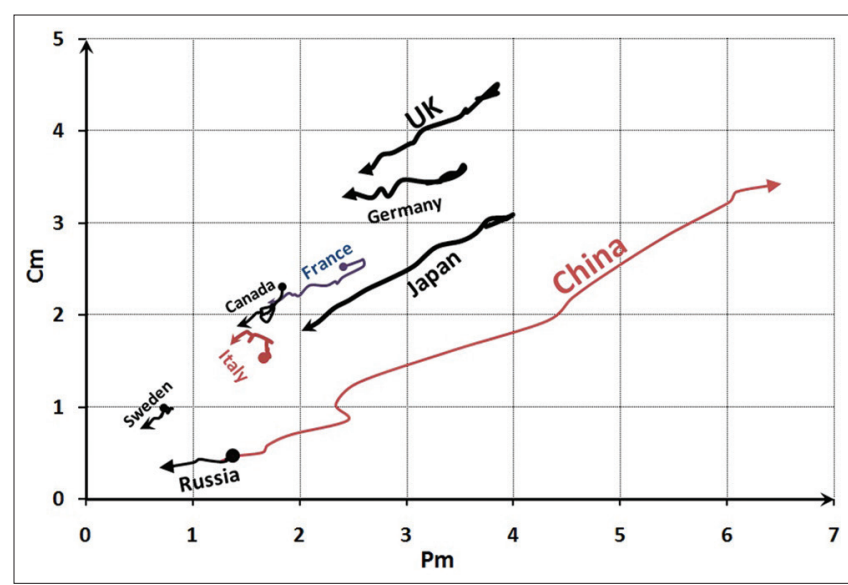

Figure 6: Two-dimensional map of countries of decline pattern. All countries of decline countries are showed except USA. China presented in sake of comparison. Italy as an exception with progress in $\mathrm{Cm}$ and decline in Pm is presented as well

time line in the map stays approximately around the same position during the specified time period, especially in comparison to the other 20 countries showing meaningful changes in their positions. Countries of "fluctuation" pattern are as follow: Argentina, Austria, Belgium, Bulgaria, Chile, Croatia, Czech Republic, Denmark, Egypt, Finland, Greece, Hong Kong, Hungary, Ireland, Israel, Mexico, The Netherlands, New Zealand, Norway, Pakistan, Poland, Portugal, Romania, Saudi Arabia, Slovakia, Slovenia, South Africa, Switzerland, Thailand and Ukraine.

Fluctuation pattern shows that these countries can manage to have the same growth rate as the average of all 50 countries, and this can be a successful achievement in comparison to the countries of decline pattern.

As for the pattern of decline, eight countries have been found to follow it, namely: Canada, France, Germany, Japan, Russian Federation, Sweden, United Kingdom and United States [Figure 4].

These countries are mostly the developed countries. This decline progress implies that these countries advancement in $P \mathrm{~m}$ and $\mathrm{Cm}$ are lagging behind the average of all
50 countries. United States acquires the fastest decline rate [Figure 2].

\section{CONCLUSION}

Based on two-dimensional approaches, first 50 of topmost countries in scientific achievement have been analyzed, and their temporal time-lines have been presented in the two-dimensional map drawn via $P \mathrm{~m}$ and $\mathrm{Cm}$. Three main groups in terms of their temporal pattern in the two-dimensional map have been recognized (namely progress, fluctuation, and decline). China has an outstanding growth rate and USA has the worst decline rate among all the countries.

\section{REFERENCES}

1. NSF, Science and Engineering Indicators (SEI), 2012. Available from: http://www.nsf.gov/statistics/seind12/c5/c5h.htm.

2. Science and Technology Indicator Project Team, NISTEP, MEXT. Japanese Science and Technology Indicators; 2011.

3. Organisation for Economic Co-operation and Development (OECD). Science, Technology and Industry Outlook; 2008.

4. European Commission, Directorate-General for Research. Science, Technology and Competitiveness, Key Figures Report; 2008/2009.

5. Vinkler P. Composite scientometric indicators for evaluating publications of research institutes. Scientometrics 2006;68:629-42.

6. Schubert A, Braun T. Relative indicators and relational charts for comparative assessment of publication output and citation impact. Scientometrics 1986;9:281-41.

7. Nejati A, Hosseini Jenab SM. A two-dimensional approach to evaluate the scientific production of countries (case study: The basic sciences). Scientometrics 2009;84:357-64.

8. Jazayeri SB, Alavi A, Rahimi-Movaghar V. Situation of medical sciences in 50 top countries from 1996 to 2010 - Based on quality and quantity of publications. Acta Med Iran 2012;50:273-8.

9. Hirsch JE. An index to quantify an individual's scientific research output. Proc Natl Acad Sci U S A 2005;102:16569-72.

10. Science and Technology Indicator Project Team, NISTEP, MEXT. Japanese Science and Technology Indicators; 2011, Chart 4-1-1.

11. Further information. Available from: http://www.scimagojr.com/ countrysearch.php.

How to cite this article: Hosseini Jenab SM. Temporal study of countries' scientific evolution based on two-dimensional approach. J Sci Res 2013;2:169-72.

Source of Support: Nil, Conflict of Interest: None declared

\section{Announcement}

\section{iPhone App}

Download

iPhone, iPad application
A free application to browse and search the journal's content is now available for iPhone/iPad. The application provides "Table of Contents" of the latest issues, which are stored on the device for future offline browsing. Internet connection is required to access the back issues and search facility. The application is Compatible with iPhone, iPod touch, and iPad and Requires iOS 3.1 or later. The application can be downloaded from http://itunes.apple.com/us/app/medknow-journals/ id458064375? $\mathrm{s}=1 \& \mathrm{mt}=8$. For suggestions and comments do write back to us. 